Objectives: To find the micro-structural correlate of the origin of bone erosions in the bare area of the human joint

Methods: Bare areas of human joints were analyzed for early microstructural changes by in-vivo high-resolution peripheral computed tomography (HR-pQCT). First, bare areas were exactly defined by scanning 6 cadaveric hands for localization of the bare area in the human metacarpal head. Bone lesions found in the cadaveric hand by HR-pQCT were additionally by super-resolution ex vivo micro-CT ( $\mu$ CT40). Then, number and distribution of the type of bare area bone lesion found in cadaveric study were analyzed in a cohort of 105 healthy individuals and 107 anti-citrullinated peptide (ACPA) positive RA patients with similar sex and age distribution.

Results: HR-pQCT combined with adaptive thresholding allowed the definition of a new type of bone lesions in the bare areas of the human joint termed "COMIC" standing for "cortical micro-channel". Their existence in the bare area was additionally validated by microCT (Figure 1). RA patients showed significantly $(p<0.001)$ more CoMiCs $(112.9 \pm 54.7 /$ joint $)$ than healthy individuals (75.2 $\pm 41.9 /$ joint) with 20-49 years old RA patients exhibiting similar CoMiC numbers as observed in over 65 year old healthy individuals. Importantly, CoMiCs were found in RA patients already very early in their disease course with enrichment in the erosion-prone radial side of the joint.
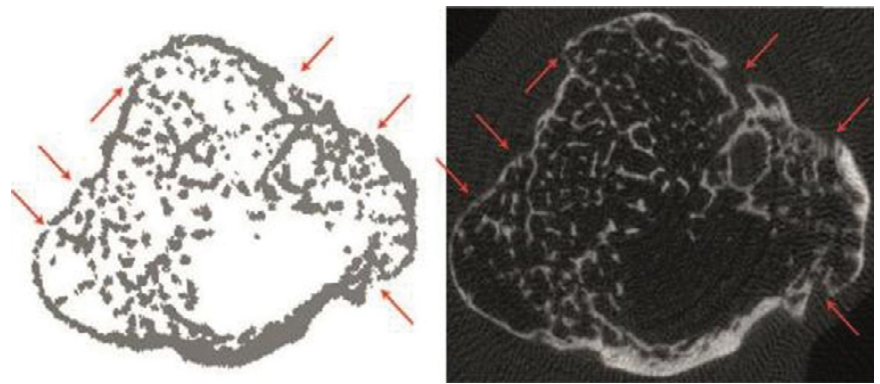

Conclusions: CoMiCs represent a new structural feature of the joint, which is characteristic for the bone of the bare area. COMICS at low level are also found in young healthy individuals but they significantly increase with age and particularly with RA. COMICs develop much earlier and much more pronounced in RA patients than in healthy individuals and therefore represent an interesting new early indicator for erosion development in ACPA positive RA patients.

References:

[1] Stach CM, Bauerle M, Englbrecht M, Kronke G, Engelke K, Manger B, et al. Periarticular bone structure in rheumatoid arthritis patients and healthy individuals assessed by high-resolution computed tomography. Arthritis \& Rheumatism. 2010;62(2):330-9.

[2] Simon D, Kleyer A, Stemmler F, Simon C, Berlin A, Hueber AJ,Haschka J, Renner N, Figueiredo C, Neuhuber W, Buder T, Englbrecht M, Rech J, Engelke K, Schett G. Age- and Sex-Dependent Changes of Intra-articular Cortical and Trabecular Bone Structure and the Effects of Rheumatoid Arthritis. J Bone Miner Res. 2016 Oct 27. doi: 10.1002/jbmr.3025. [Epub ahead of print]

Disclosure of Interest: None declared

DOI: 10.1136/annrheumdis-2017-eular.3730

\section{SAT0629 ELECTRODIAGNOSTIC VS ULTRASONOGRAPHY: WHICH ONE IS BETTER TO CONFIRM DIAGNOSIS OF ULNAR NEUROPATHY AT ELBOW?}

S.M. Rayegani ${ }^{1}$, E. Loni ${ }^{2}$, S.A. Raeissadat ${ }^{3}$, E. Kargozar ${ }^{4}$, S. Rahimi Dehgolan ${ }^{1}$. ${ }^{1}$ Physical Medicine and Rehabilitation Research Center, Shahid Beheshti University of Medical Sciences; ${ }^{2}$ Rofeideh rehabilitation Hospital, University of Social Welfare and Rehabilitation Sciences; ${ }^{3}$ Physical Medicine and Rehabilitation Research Center, ShahidBeheshti University of Medical Sciences; ${ }^{4}$ Physical Medicine and Rehabilitation, Baharloo Hospital, Faculty of Medicine, Tehran University of Medical Sciences, Tehran, Iran, Islamic Republic Of

Background: Ulnar neuropathy at the elbow is the second most common compression neuropathy preceded by carpal tunnel syndrome. Although this diagnosis has been traditionally confirmed by electrodiagnosis (EDX), ultrasonography (US) is a re-emerging alternative method which can also evaluate the cubital zone anatomy. This study determines the maximum amount of US sensitivity and specificity by assessing different sonographic parameters and evaluates consistency and diagnostic value of the best US method in compare with EDX.

Methods: We included 66 participants (32 elbows of patient and 34 normal elbows) and performed physical exam, US and EDX for both groups. Patients were classified into four severity grades using EDX criteria. The parameters of US were cross sectional area (CSA) of ulnar nerve at three levels: medial epicondyle (CSA med), $2 \mathrm{~cm}$ distal (CSA dist) and $2 \mathrm{~cm}$ proximal (CSA prox) to medial epicondyle. Then we would be able to evaluate consistency between two tests using area under receiver operating curve (AU-ROC) method and also to determine the optimum CSA cut-off point to better diagnosis of ulnar neuropathy by US.

Results: Our findings showed that CSA med and CSA dist had significantly larger size in patients compared to normal participants ( $\mathrm{P}$-value $=0.01$ and 0.05 respectively). This increase in nerve size was more prominent among those who had axonal lesion rather than patients with demyelinated lesion ( $p$-value $<0.01$ ) Moreover those who had longer duration of symptoms had significantly higher CSA med. and CSA dist. ( $p$-value $=0.015$ and 0.001 respectively). The other promising findings were two important points; First a strong correlation between CSA med. and severity grade $(\mathrm{p}$-value $=0.034)$ and the second correlation was between CSA med and CSA dist. with a cross-elbow nerve conduction velocity (NCV) ( $p$-value $=0.01$ and 0.02 , respectively). Finally we assessed US diagnostic value as it showed $\mathrm{AU}-\mathrm{ROC}=0.871$, that means a very good coverage for an alternative diagnostic method. Also our results showed specificity of $80 \%$ and sensitivity of $84 \%$ for US in the CSA med cut-off point $=9 \mathrm{~mm}^{2}$ for diagnosis of ulnar nerve entrapment at elbow.

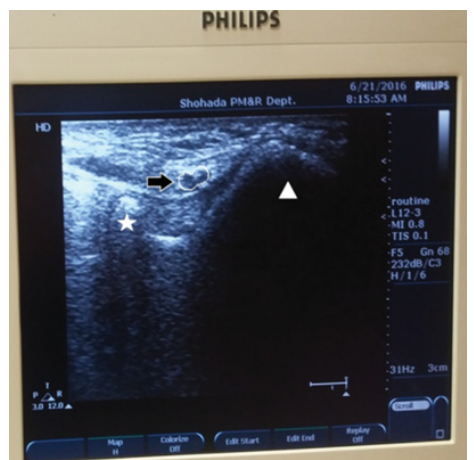

Conclusions: Based on these results we can conclude that US is a highly sensitive and specific method to diagnose ulnar neuropathy at elbow and can be used as an alternative and complementary method in diagnosis of ulnar neuropathy at elbow in particular when EDX is not available. However it could not be still a definitive and substitute mutually exclusive method to EDX in diagnosis of ulnar neuropathy

Acknowledgements:

Disclosure of Interest: None declared

DOI: 10.1136/annrheumdis-2017-eular.1694

\section{SAT0630 EXTRA-ARTICULAR MUSCULOSKELETAL INVOLVEMENT IN JUVENILE IDIOPATHIC ARTHRITIS: CLINICAL AND ULTRASONOGRAFIC FINDINGS}

V. Ravagnani ${ }^{1}$, F. Licciardi $^{2}$, D. Montin ${ }^{2}$, F. Santarelli $^{3}$, S. Martino ${ }^{4}$, C. Cirone ${ }^{5}$ ${ }^{1}$ Internal Medicine, ASST MANTOVA - C. Poma Hospital, Mantova; ${ }^{2}$ ImmunoRheumatology Unit, Regina Margherita Children Hospital Città della Salute e della Scienza; ${ }^{3}$ ImmunoRheumatology Unity, Regina Margherita Children Hospital, Città della Salute e della Scienza; ${ }^{4}$ ImmunoRheumatology Unity, Regina Margherita Children Hospital Città della Salute e della Scienza; ${ }^{5}$ ImmunoRheumatology Unit, Regina Margherita Children Hospital, Città della Salute e della Scienza, Torino, Italy

Background: in Juvenile Idiopathic arthritis (JIA), musculoskeletal ultrasound (MSUS) has been proven to be more sensitive than clinical evaluation in detecting articular synovitis. Nevertheless, many studies report a variable percentage of clinically active joints, that are judged normal by ultrasound examination. In absence of a feasible and reliable gold standard for pediatric synovitis (histology or MRI), this point may weaken the confidence in ultrasound, that is nevertheless perceived as an interesting tool, in the management of JIA.

Objectives: This preliminary study investigates the possibility that sometimes the clinically detected synovitis could be missed by ultrasound, because of its extra-articular localization.

Methods: 43 consecutive children affected by JIA underwent separated clinical and ultrasound assessments, blindly, in the same day. Patients were followed up in a pediatric Rheumatology Unit. The following clinical data were collected: age, sex, disease duration, subset of JIA, ongoing therapy, previous therapy, disease activity. By MSUS, the synovitis was investigated bilaterally, both in gray scale and power Doppler, in the MCP and subtalar joints, wrists, knees, ankles, in the flexor and extensor tendons of the wrist and hand, in the anterior, medial and lateral tendons of the ankle, in the synovial bursae of knee and ankle. The possible involvement of the entheses was also investigated. The definition of ultrasonographic synovitis was based on the preliminary OMERACT definitions of synovitis in children. The inter and intra observer reproducibility of the MSUS examination was tested, independently, both between two operators and through a second assessment of the stored images.

Results: 43 children affected by JIA were recruited, in the outpatient clinic of the Regina Margherita Pediatric Hospital of Torino, Italy. They were 9 boys and 34 girls, median age 7,7 (IQR 5,5-10,1), 27 oligoarticular, 11 poliarticular, 4 psoriatic arthritis, 1 undifferentiated arthritis. The median disease duration was 44 months (IQR: 20,5-61,5), 20 patients in remission, 23 with active disease. 774 joints, 1548 synovial sheaths, 430 entheses and 258 synovial bursae were assessed. The physical examination detected inflammation in 54 joints, 33 tendons, 0 entheses, 